\title{
ERRATUM
}

Yan-Ling Qiu · Yuji Sekiguchi · Hiroyuki Imachi Yoichi Kamagata $\cdot$ I-Cheng Tseng $\cdot$ Sheng-Shung Cheng Akiyoshi Ohashi · Hideki Harada

\section{Sporotomaculum syntrophicum sp. nov., a novel anaerobic, syntrophic benzoate-degrading bacterium isolated from methanogenic sludge treating wastewater from terephthalate manufacturing}

Published online: 29 April 2003

(C) Springer-Verlag 2003

\section{Arch Microbiol (2003) 179:242-249}

The JCM (Japan Culture Collection) number for the strain FB Sporotomaculum syntrophicum described in the paper is wrong. The number should be JCM 11495.

The online version of the original article can be found at http://dx.doi.org/10.1007/s00203-003-0521-z

Y.-L. Qiu · H. Imachi · A. Ohashi · H. Harada Department of Environmental Systems Engineering, Nagaoka University of Technology,

940-2188 Nagaoka, Niigata, Japan

Y. Sekiguchi $(\bowtie) \cdot$ Y. Kamagata

Microbial and Genetic Resources Research Group, Research Institute of Biological Resources,

National Institute of Advanced Industrial Science and Technology (AIST), Tsukuba Central 6,

1-1 Higashi 1, 305-8566 Tsukuba, Ibaraki, Japan

Tel.: +81-298-616590, Fax: +81-298-616587,

e-mail: y.sekiguchi@aist.go.jp

I-C. Tseng

Department of Biology, National Cheng Kung University, Tainan 701, Taiwan

S.-S. Cheng

Department of Environmental Engineering,

National Cheng Kung University, 701 Tainan, Taiwan 